\title{
Cobertura vacinal para Hepatite $B$ na Amazônia brasileira e os riscos à transmissão vertical
}

\author{
Vaccine coverage for Hepatitis B in the Brazilian Amazon and risks to vertical transmission \\ Cobertura vacunal para Hepatitis B en la Amazonia brasileña y los riesgos a la transmisión vertical
}

Recebido: 10/06/2021 | Revisado: 19/06/2021 | Aceito: 28/06/2021 | Publicado: 11/07/2021

Carlos Arthur Holanda Figueiras Pereira

ORCID: https://orcid.org/0000-0002-5449-9009

Centro Universitário Metropolitano da Amazônia, Brasil

E-mail: carlos.arthurhfp@outlook.com

Iuri Santiago Leão de Sales

ORCID: https://orcid.org/0000-0001-6391-617X

Centro Universitário Metropolitano da Amazônia, Brasil

E-mail: iuri.sales@hotmail.com

Jamylle Pedrosa Gomes

ORCID: https://orcid.org/0000-0002-4273-7663

Centro Universitário Metropolitano da Amazônia, Brasil

E-mail: jamyllepedrosa@ hotmail.com

Kamylle Pedrosa Gomes

ORCID: https://orcid.org/0000-0003-1579-3982

Centro Universitário Metropolitano da Amazônia, Brasil

E-mail: kamyllepgomes@outlook.com

Luiza Bastos Campos

ORCID: https://orcid.org/0000-0002-2949-3414

Centro Universitário Metropolitano da Amazônia, Brasil

E-mail: luizabastoscampos@gmail.com

Marlon Vaz da Rocha

ORCID: https://orcid.org/0000-0002-2733-7434

Centro Universitário Metropolitano da Amazônia, Brasil

E-mail: marlonrocha28@gmail.com

Murilo Mendonça Aguiar

ORCID: https://orcid.org/0000-0002-4438-9578

Centro Universitário Metropolitano da Amazônia, Brasil

E-mail: muriloaguiarmed@outlook.com

Sarah Jennyfer Lima Lopes

ORCID: https://orcid.org/0000-0002-8121-5761

Centro Universitário Metropolitano da Amazônia, Brasil

E-mail: sarahjennyfer20@gmail.com

Vitória Amaral Lima

ORCID: https://orcid.org/0000-0002-6248-9580

Centro Universitário Metropolitano da Amazônia, Brasil

E-mail: vitoriaamarallima18@gmail.com

Sergio Beltrão de Andrade Lima

ORCID: https://orcid.org/0000-0002-9531-2482

Centro Universitário Metropolitano da Amazônia, Brasil

E-mail: sergio.lima@autlook.com

Maria Helena Rodrigues de Mendonça

ORCID: https://orcid.org/0000-0003-0571-0565

Centro Universitário Metropolitano da Amazônia, Brasil

E-mail: sergio.lima@autlook.com

\section{Resumo}

Objetivo: Avaliar a cobertura vacinal para Hepatite B e Pentavalente na Amazônia brasileira e os riscos à transmissão vertical do Vírus da Hepatite B, período de 2010 a 2019. Metodologia: Estudo observacional, transversal e descritivo, da série temporal da cobertura vacinal de rotina para Hepatite B e Pentavalente, Taxa de Detecção da hepatite B em gestantes por 100mil nascidos vivos e idade gestacional, percentual da assistência ao Pré-Natal e Taxa de Detecção da hepatite B na infância por 100mil habitantes e suas formas clínicas. Resultados: Foi observada baixa cobertura vacinal em ambas as vacinas, com exceção apenas de Rondônia/RO para Hepatite B, com média de 103,5\% e homogeneidade de 88,5\%; para Pentavalente, houve máxima em RO $(91,6 \%)$ e mínima no Pará $(63,5 \%)$, com heterogeneidade municipal. Houve registro de casos de hepatite B em gestante em todos os anos e estados, com maior diagnóstico no $3^{\circ}$ trimestre considerando a região Norte no geral, com assistência frequente em "Mais que adequada" (máxima de 
$61 \%$ ), contudo, ressalta preocupação às que não realizaram assistência e/ou apresentaram inadequações. Na infância, se destaca menores de 1 ano (45,5\%/100.000), na forma crônica (45,1\%). Conclusão: Foi observada baixa cobertura vacinal para ambas as vacinas, falha na homogeneidade das coberturas e casos de hepatite B em gestantes com frequência ao diagnóstico no terceiro trimestre e assistência ao Pré-Natal inadequada em diferentes ocasiões, portanto, aumento os riscos de transmissão vertical e os riscos de cronicidade ao recém-nascido.

Palavras-chave: Cobertura vacinal; Hepatite B; Vírus da Hepatite B; Vigilância epidemiológica.

\begin{abstract}
Objective: To evaluate the vaccine coverage for Hepatitis B and Pentavalent in the Brazilian Amazon and the risks to vertical transmission of Hepatitis B virus, period from 2010 to 2019. Methodology: An observational, cross-sectional, and descriptive study of the time series of routine vaccination coverage for Hepatitis B and Pentavalent, Hepatitis B Detection Rate in pregnant women per 100,000 live births and gestational age, percentage of pre-pregnancy care Natal and Hepatitis B Detection Rate in childhood per 100,000 inhabitants and their clinical forms. Results: Low vaccination coverage was observed in both vaccines, except only for Rondônia/RO for Hepatitis B, with an average of $103.5 \%$ and homogeneity of $88.5 \%$; for Pentavalente, there was a maximum in OR $(91.6 \%)$ and a minimum in Pará $(63.5 \%)$, with municipal heterogeneity. There was a record of cases of hepatitis B in pregnant women in all years and states, with higher diagnosis in $3^{\circ}$ trimester considering the North region in general, with frequent assistance in "More than adequate" (maximum 61\%) however, concerns are highlighted for those who did not receive assistance and/or showed inadequacies. In childhood, it stands out younger than 1 year $(45.5 \% / 100,000)$, in the chronic form $(45.1 \%)$. Conclusion: Low vaccination coverage was observed for both vaccines, failure in homogeneity of coverage and cases of hepatitis B in pregnant women with frequency of diagnosis in the third trimester and inadequate prenatal care in different occasions, therefore, increased risks of vertical transmission and risks of chronicity to the newborn.
\end{abstract}

Keywords: Vaccine coverage; Hepatitis B; Hepatitis B virus; Epidemiological surveillance.

\title{
Resumen
}

Objetivo: Evaluar la cobertura vacunal para Hepatitis B y Pentavalente en la Amazonia brasileña y los riesgos a la transmisión vertical del Virus de la Hepatitis B, período de 2010 a 2019. Metodología: Estudio observacional, transversal y descriptivo, de la serie temporal de la cobertura vacunal de rutina para Hepatitis B y Pentavalente, Tasa de Detección de la hepatitis B en gestantes por 100mil nacidos vivos y edad gestacional, porcentual de la asistencia al Pre-Natal y Tasa de Detección de la hepatitis B en la infancia por 100.000 habitantes y sus formas clínicas. Resultados: Se observó baja cobertura vacunal en ambas vacunas, con excepción solo de Rondônia/RO para Hepatitis B, con promedio de 103,5\% y homogéneneneidad de 88,5\%; para Pentavalente, hubo máxima en RO (91,6\%) y mínima en Pará (63,5\%), con heterogeneidad municipal. Hubo registros de casos de hepatitis B en gestante en todos los años y estados, con mayor diagnóstico en el $3^{\circ}$ trimestre considerando la región Norte en general, con asistencia frecuente en "Más que adecuada" (máxima del 61\%)Sin embargo, resalta preocupación a las que no realizaron asistencia y/o presentaron inadaptaciones. En la infancia, se destaca menores de 1 año (45,5\%/100.000), en la forma crónica $(45,1 \%)$.Conclusión: Se observó baja cobertura vacunal para ambas vacunas, falla en la homogéneneneidad de las coberturas y casos de hepatitis B en gestantes con frecuencia al diagnóstico en el tercer trimestre y asistencia al Prenatal inadecuada en diferentes ocasiones, Por lo tanto, aumenta los riesgos de transmisión vertical y los riesgos de cronicidad al recién nacido.

Palabras clave: Cobertura vacunal; Hepatitis B; Virus de la Hepatitis B; Vigilancia epidemiológica.

\section{Introdução}

As hepatites virais são patologias ocasionadas por distintos agentes etiológicos e características epidemiológicas, clínica e laboratoriais específicas, contudo, com tropismo aos hepatócitos (Gonçalves et al., 2019; Brasil, 2020). Precisamente sobre o vírus da Hepatite $\mathrm{B}$ (VHB), pertence à família Hepadnaviridae, existente em caráter mundial, é considerado um problema de saúde pública devida sua alta transmissibilidade, principalmente por via sexual, parenteral e vertical, podendo estar relacionado às diferentes características socioeconômicas da população (Brasil, 2019).

Segundo a Organização Mundial da Saúde (2018), estima-se que cerca de 350 milhões de pessoas são portadoras do VHB, e que haja mais de 780 mil óbitos anualmente sendo, portanto, considerada a décima principal causa de morte e, em nível de Brasil, segundo dados do Boletim Epidemiológico (2020), de 1999 a 2019, foram confirmados 247.890 casos de hepatite B e, cerca de $0,52 \%$ (1,1milhão) são portadores crônicos da doença.

Referente ao estado de cronicidade, em adultos, de $90 \%$ a $95 \%$ evoluem a cura e $5 \%$ a $10 \%$ permanecem como reagente $(\mathrm{HBsAg+})$ por mais de seis meses, logo, fundamenta-se a forma clínica crônica da hepatite B. De acordo com OMS 
(2018) e Gonçalves et al (2019), apenas um a cada 20 infectados possuem conhecimento sobre seu estabelecimento, logo, implicando nas medidas de tratamento e favorecendo a evolução às formas graves com surgimento de cirrose e hepatocarcinoma, nesse cenário, aumentam as necessidades de transplantes de fígado (OMS, 2018).

Dentre todas as formas de transmissão, merece destaque a transmissão vertical (mãe para filho) que, embora a doença seja um problema em todas as faixas etárias, o recém-nascido tem cerca de $90 \%$ de chances de se tornar crônico e $25 \%$ podem evoluir a óbito caso não seja realizado a imunoprofilaxia adequada que consiste, sobretudo, na aplicação da imunoglobulina humana Anti-hepatite B e a vacina Hepatite B (Francisco et al., 2015).

Com isso, durante o período gestacional, é extremamente importante o acompanhamento da gestante ao Pré-Natal como forma de triagem à hepatite B a partir da realização de teste HBsAg com objetivo de fundamentar as ações de prevenção à transmissão vertical seja por meio da imunização da gestante ou o tratamento oportuno. O estabelecimento de doenças durante a gestação gera consequências e aumentam as morbidades, como a prematuridade e o baixo peso (Sanson et al., 2018; Souza et al., 2019). Destarte, como base para evitar esse cenário de vulnerabilidade entre a população, sobretudo, na infância, as vacinas emergem como estratégia extremamente eficaz e segura frente à diminuição da morbimortalidade (Brasil, 2020).

Desde o século passado, as ações de imunização adjunta a vigilância epidemiológica são responsáveis pelas maiores conquistas em nível mundial, como exemplo da erradicação da varíola e eliminação da poliomielite em diversos países a partir da vacinação em massa e o alcance da imunização de rebanho (Dias e Almeida, 2017; Fiocruz, 2020). Contudo, todo esse sucesso não seria alcançado sem um alicerce estratégico e fundamentado em uma ótica de incentivo à modernização tecnológica, logo, define-se o Programa Nacional de Imunização (PNI), criado em 1973 pelo Ministério da Saúde - MS, mundialmente conhecido como coordenador das avaliações, planejamento e monitoramento das ações de imunização, das coberturas vacinais, infraestrutura e logística dos imunobiológicos, sendo esses oferecidos de forma gratuita e rotineiramente nos serviços de saúde, com intuito de atingir e manter adequas às metas das coberturas estabelecidas pelo MS (Brasil, 2020; Chaves et al., 2020).

Nesse sentido, seguindo as diretrizes do Sistema Único de Saúde (SUS), o PNI estabelece o Calendário Nacional de Vacinação com a oferta de mais de 19 imunobiológicos distribuídos em todas as faixas etárias e, dentre elas, destacam-se a vacina da Hepatite B inativada, com cobertura vacinal preconizada de 95\% e esquema vacinal na rotina composto por 3 doses ( 0,1 e 6 meses). Contudo, é ofertado nas unidades de saúde para todas as faixas etárias, especialmente para gestantes não imunizadas devendo, portanto, seguir para dosagem do anti-HBs como meio de monitorar o êxito da vacinação, uma vez que a resposta à vacina depende de cada organismo. Não obstante, é altamente eficaz com prevenção em mais de $90 \%$ dos adultos jovens e em mais de 95\% em lactentes, crianças e adolescentes, com baixa frequência de eventos adversos (SBIm, 2020).

Em 2012, como intuito de minimizar o quantitativo de injeções em um mesmo momento, a vacina Pentavalente, que previne difteria, tétano, coqueluche, hepatite B e meningites causadas pelo Haemophilus influenzae tipo b, foi introduzida ao PNI e, desde então, é administrada em lactantes nas doses subsequentes à primeira dose com Hepatite $\mathrm{B}$, sendo também preconizados 95\% de cobertura, possuindo alta imunogenicidade (Brasil, 2012; SBIm, 2020).

Destarte, embora a eficácia das vacinas seja enormemente relatada na literatura, à conduta frente não adesão às vacinas torna-se um problema às coberturas vacinais e à saúde pública sendo, portanto, influenciado por diversos fatores como a falta de acesso à informação em saúde e a relevância da vacinação, baixa percepção de riscos e consequências das doenças, desinformação acerca da eficácia e segurança das vacinas, subfinanciamento do SUS e desabastecimento dos imunobiológico. Contudo, independente dos fatores, o aumento na recusa vacinal fundamenta o perfil de susceptibilidade na população e favorece as emergências em saúde pública e o estabelecimento de doenças imunopreveníveis.

Logo, sendo a hepatite B um enorme problema à sociedade, sobretudo na infância, as avaliações constantes das coberturas vacinais são fundamentais para subsidiar ações pontuais e focalizadas em uma determinada área, além disso, sendo 
os recém-nascidos os mais vulneráveis às formas graves da doença e a transmissão vertical um grande potencial de risco, torna-se essencial estabelecer um contexto de assistência à gestante e os riscos à transmissão.

Portanto, o estudo tem por objetivo avaliar a cobertura vacinal para Hepatite B e Pentavalente na Amazônia brasileira e os riscos à transmissão vertical do Vírus da Hepatite B, período de 2010 a 2019.

\section{Metodologia}

Trata-se de um estudo observacional, de aspecto transversal, com caráter descritivo e analítico abordando uma análise de séries temporais da Cobertura Vacinal de rotina para Hepatite B e Pentavalente, além da Taxa de Detecção da hepatite B em gestantes por 100.000 nascidos vivos, a frequência percentual da idade gestacional e indicador de assistência ao Pré-Natal na região Norte, no período de 2010 a 2019 e, Taxa de Detecção da hepatite B na infância, segundo a forma clínica da doença, no período de 2010 a 2018.

Os dados referentes às Coberturas Vacinais (CV) foram obtidos através do Sistema de Informação do Programa Nacional de Imunizações (SI-PNI/MS), no período 2010 a 04/09/2019 e utilizou-se o cálculo:

$$
\mathbf{C V}=\frac{\text { Quantitativo de imunobiológico aplicados em }<1 \text { ano (população alvo) }}{\text { Quantitativo populacional }(<1 \text { ano })} \times 100
$$

Seguindo PNI, esse cálculo é realizado somando as 3 doses da vacina Hepatite B e, separadamente, as 3 doses da Pentavalente, logo, coberturas distintas. A partir disso, foi obtida a média das coberturas entre estados e municípios e então, considerando a cobertura preconizada pelo MS de $95 \%$ e, posteriormente, foram identificados os estados e municípios que apresentaram baixa cobertura.

Para estabelecer os casos de hepatite B em gestantes e na infância, os dados foram extraídos do Departamento de Informática do Sistema Único de Saúde (DATASUS/MS) para cada estado e ano, sendo a atualização dos dados em 31/12/2019. A Taxa de Detecção (TA) para gestantes foi padronizada de acordo com o quantitativo de nascidos vivos, sendo calculado pela fórmula:

$$
\mathbf{T A}=\frac{\text { Quantitativo de casos de hepatite B em gestantes por ano (2010 a 2019) }}{\text { Quantitativo de nascidos vivos }} \times 100.000
$$

A partir desse cálculo, foi obtido a Taxa de Detecção na infância ( $<1$ a 14 anos), considerando o quantitativo de casos de 2010 a 2018 como numerador e quantitativo de habitantes como denominador x 100.000.

Para estabelecer os dados da idade gestacional, assistência ao Pré-Natal e a forma clínica de ocorrência na infância, foram obtidos pelo DATASUS sendo expresso em frequência relativa sob forma de tabelas.

Para formação de gráficos e tabelas, foi utilizado o programa Microsoft Excel 2016. Os dados foram coletados e registrados em dados quantitativos que representam o perfil da cobertura vacinal de acordo com os seguintes fatores: Média da Cobertura vacinal por Unidade Federativa e municípios no Norte, e casos de hepatite B em gestantes e crianças (HBsAg positivo) por local de residência, segundo variáveis de estudo (idade gestacional, indicador de assistência ao Pré-Natal, faixa etária infantil e forma clínica da doença).

\section{Resultados e Discussão}

A partir dos dados obtidos pelo Sistema de Informação do Programa Nacional de Imunizações (SI-PNI/MS) para cada 
Unidade Federativa (UF) da região Norte, no período de 2010 a 2019, considerando a média da Cobertura Vacinal (CV) na rotina para vacina da Hepatite B, foi observado cobertura preconizada apenas para Rondônia/RO (103,5\%), com baixa cobertura para demais Unidades Federativas, sendo elas: Tocantins/TO (94,8\%), Amazônia/AM (89\%), Roraima/RR (87,1\%), Acre/AC (81,2\%), Pará/PA (80\%) e Amapá/AP (79,9\%) (Figura 1/A). Na análise municipal, apenas RO alcançou a homogeneidade (>75\%), com 88,5\% dos municípios apresentando CV ideal, e os demais, TO 68,3\% dos municípios, AP (37,5\%), AM (30,6\%), RR (26,7\%), PA (21,5\%), AC (4,5\%) não atingiram a CV ideal (Figura 1/B). Referente à Pentavalente, todos os estados encontraram-se abaixo do ideal, RO com 91,6\%, TO (83,4\%), RR (78,6\%), AM (76,2\%), AC (67,3\%), AP $(67,2 \%)$ e PA (63,5\%), e baixa cobertura em 84,4\% dos municípios (Figura 1/C; D).

Figura 1 - Média da Cobertura vacinal (CV) em menores de 1 ano na região Norte. Onde A, B: distribuição da CV para Hepatite B entre os estados e municípios, no período de 2010 a 2019; C, D: distribuição da CV para Pentavalente entre os estados e municípios, de 2012 a 2019.

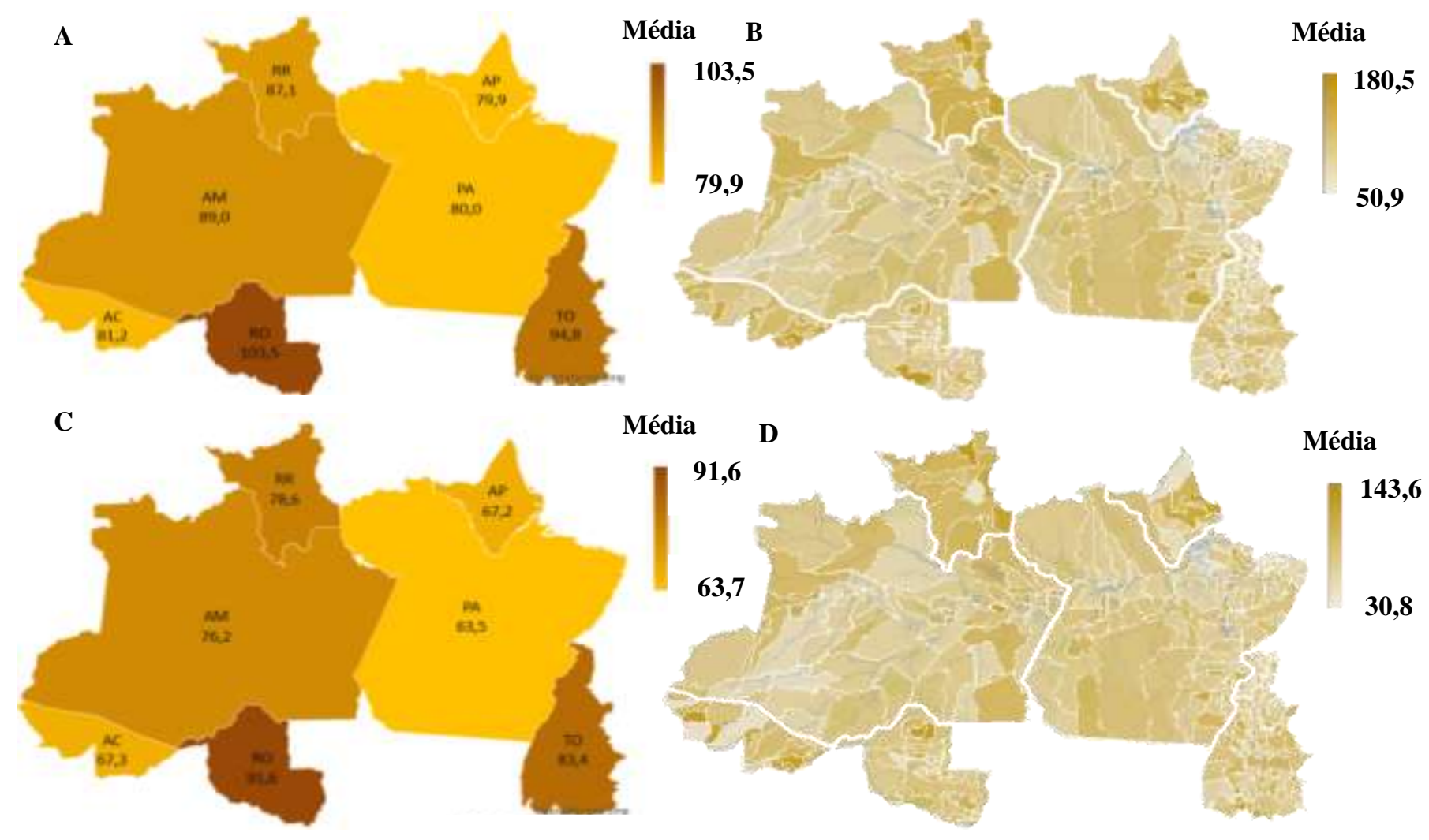

Fonte: Sistema de Informação do Programa Nacional de Imunizações - SI-PNI (atualização dos dados: 04/09/2019).

A partir dos dados, fica evidente a baixa vacinação no público inferior a 1 ano para ambas as vacinas, além da visível heterogeneidade das coberturas entre os municípios. Nesse contexto, precisamente sobre a vacina Hepatite $\mathrm{B}$, apesar dos dados do SI-PNI disponibilizarem cobertura vacinal somando as 3 doses e, portanto, impossibilitar a quantificação da taxa de abandono, Maciel et al (2019) expõe que $90 \%$ das crianças recebem a primeira dose ao nascer, entretanto, apenas 2,2\% tornam-se imunizadas devido a alta taxa de abandono às doses subsequentes. No mais, nesse contexto, as justificativas cabíveis envolveriam a introdução da vacina Pentavalente ao esquema vacinal à prevenção da doença, contudo, cerca de 1 a cada 4 crianças não a recebem sendo, portanto, condizente com os dados expostos nesse estudo, onde ambas não atingiram a meta de 95\%, assim, o público infantil apresenta alto risco de infeção pelo vírus da hepatite B e às outras doenças preveníeis pela Pentavalente (Macedo et al., 2017). 
O sucesso do PNI é avaliado a partir do alcance das coberturas e a homogeneidade dos municípios o qual cria um ambiente propício à chamada "imunidade de rebanho", entretanto, os dados coletados demonstram certo distanciamento aos objetivos do programa. No mais, esse cenário não é restrito às vacinas que previnem à hepatite B, segundo Pinheiro (2020), todas as vacinas administradas na infância encontram-se abaixo da meta preconizada pelo Ministério da Saúde e, até 2019, o percentual médio da cobertura vacinal dos 19 imunobiológicos presentes no calendário infantil estava em 51,5\%, sendo a máxima de 59,7\% para o primeiro reforço da Tríplice Bacteriana (DTP) e mínima de 19,9\% para Tetra Viral, sendo recomendados 95\% para ambas, expondo uma piora nesse cenário no ano de 2020 (Macedo et al., 2017; Chaves et al., 2020; SBIm, 2020).

Para Wolkers et al (2016), vacinação na infância é um direito a vida e precisa ser respeitado e assegurado, devendo todas as crianças ter acesso igualitário, contudo, tal conjuntura apresenta-se entraves e, em sua maioria, é subestimada por muitos pais. Para Fonseca e Buenafuente (2018), a vacinação em menores de um ano encontra-se abaixo do preconizado e com maiores taxas de abandono das doses. Para Silva et al (2018) e Silva et al (2020), a incompletude vacinal é um dos principais problemas à saúde pública estando presente, sobretudo, na faixa de 25 a 35 meses com mães de baixa renda, sem plano de saúde e com dificuldade ao acesso dos serviços de saúde, onde o abandono chega a 48,9\% para Pneumocócica 10 valente, Meningocócica C (32,2\%), Rotavírus Humano (18,8\%), Triplice Viral (10,6\%), Febre Amarela (9,5\%), Tetravalente (7,9\%) e Hepatite B $(5,8 \%)$.

Destarte, indo a consenso, Castro et al (2018) expõe a vacinação em menores de dois anos não adequa tanto no primeiro quanto no segundo ano de vida, principalmente em mães com menor recurso financeiro e nível educacional considerado baixo $\left(>9^{\circ}\right.$ ano), portanto, sendo justificado pelos autores devido as limitações logísticas no acesso aos transportes, e o menor grau de instrução quanto aos benefícios da vacinação e completude do esquema vacinal. É fato a enorme influência desses fatores e sua relação estritamente discutida na literatura, contudo, outros autores apresentam argumentos controversos.

Maciel et al (2019) e Silveira et al (2020), apresentam que mães com maior nível de instrução e maior acumulo de renda apresentam menores adequações na caderneta infantil, logo, esses dados remetem a não vacinação como um caráter multifatorial, complexo e com grande contribuição à incidência e reincidência de doenças, sobretudo, na infância devido ao alto grau de vulnerabilidade e susceptibilidade e, consequentemente, agravando as preocupações em nível de saúde pública coletivo, como exposto recentemente pelo surto do sarampo no Brasil responsável por 10.498 casos no período de Fevereiro de 2018 a Junho de 2019, com maiores percentagem nos estados da região Norte (AM/93,4, PA/3,4 e RR/1,5), em todas as faixas etária, entretanto, majoritariamente em menores de 4 anos (Tauil et al., 2017; Chaves et al.,2020).

Para Nóvoa et al (2020), em uma análise temporal inter-regional da cobertura aos imunos do calendário vacinal, de 1994 a 2019, esse perfil de decréscimo vem sendo exposto, sequencialmente, desde 2016, com maior visibilidade na região Norte e Nordeste, regiões de menor Índice de Desenvolvimento Humano, com 0,667 e 0,663, respectivamente, todavia, Maciel et al (2019), expõem não haver uma relação positiva entre o indicador IDH e a cobertura vacinal, uma vez que as taxas de inadequação do cartão vacinal encontram-se em valores aproximados em áreas de maior e menor IDH indo, portanto, em consenso ao Césare (2020) e Silva et al (2020), onde a baixa adesão a vacina não é restrita, sobretudo, em bases econômicas e sim na problemática relação entre o individuo para com a coletividade que, em sua maioria, utilizam da tão relatada liberdade de escolha em direção a não adesão as vacinas e, assim, omitem esse ato como uma responsabilidade social e sanitária.

Entretanto, é valido expor a esse posicionamento a existência da desinformação referente à eficácia e segurança das vacinas, complexidade do calendário de vacinação a partir da introdução de novas vacinas e os esquemas vacinais, desabastecimento pontuais dos imunobiológicos, restrições de acesso às vacinas e o subfinanciamento do SUS. Na vacinação, embora a proteção seja individual, a incansável busca pelos níveis adequados da cobertura vacinal é crucial para o alcance da 
prevenção coletiva e ao controle do estado saúde da população, logo, é cada vez mais necessária uma força conjunta dos entes governamentais e comunidade científica para com a sociedade em busca de soluções que proporcione um fortalecimento das informações, um serviço de qualidade e com acesso universal.

Para Organização Mundial da Saúde - OMS (2014), a hesitação vacinal é caracterizada como a recusa ou atraso na admissão da vacina, logo, diante aos fatores, não minimizando a influência aos demais e precisamente sobre a vacina Hepatite B aplicada nas primeiras 24 horas de vida, fundamenta a problemática diante a disponibilidade que, de acordo com Prado (2015) e Souza (2020) encontram-se em baixo estoque e com distribuição intermitente em diferentes estados, visto isso, em nota, a SBIm (2015) preconiza a utilização da Pentavalente como cumprimento ao esquema vacinal, contudo, Pinheiro (2020) expõem irregularidade no fornecimento vacina e desabastecimento nos postos de saúde em todo Brasil, tendo como principal justificativa problemas com laboratórios externos fornecedores do insumo e inadequação do produto após teste de qualidade do Instituto Nacional de Controle de Qualidade em Saúde (INCQS) pela Agência Nacional de Vigilância Sanitária (Anvisa).

Pinheiro (2020) levanta tal precariedade dos insumos à falta de políticas de incentivo e inovação na produção interna uma vez que, segundo Agencia Brasil, o país não possui um laboratório produtor interno, portanto, depende do Fundo Estratégico da Organização Pan-Americana da Saúde (OPAS), nesse contexto, remete a importância de ter uma infraestrutura adequada para produção local, aumentando a possibilitada de atuar de forma autônoma com gestão adequada e diminuindo fatores que interfere na qualidade.

Ademais, outro ponto levantado por Pinheiro (2020), indo a consenso Cruz (2017), é o modelo de atenção voltado às medidas curativas de condições agudas e doenças pré-estabelecidas, visto que o país manteve um incentivo fiscal de 11 bilhões na indústria farmacêutica somente em 2018 e, em 2020, reduziram o orçamento em mais de 300 milhões da Fundação Oswaldo Cruz - Fiocruz, sendo os laboratórios públicos Biomanguinhos e Instituto Butantã, de patrimônio nacional, os principais contribuintes à manutenção da saúde da população brasileira. Logo, fatores intrínsecos as dificuldades em estabelecer infraestrutura necessária para preparação própria de vacinas, aumentando a probabilidade de reemegência e reincidência de doenças imunopreveníveis.

A vacinação está disposta em um sistema de saúde como atividade da Atenção Básica, fundamentando nos princípios da universalidade e equidade do Sistema Único de Saúde, contudo, não é o observado. Diante disso, a Sociedade Brasileira de Pediatria (2020) se posiciona ao desabastecimento de vacinas nos postos públicos de vacinação e demonstram preocupações frente à indisponibilidade dos insumos nos últimos anos em mais de 37 mil salas de vacinação públicas do país e, sendo a imunização uma intervenção altamente eficaz para erradicação, controle e prevenção das morbimortalidade ocasionadas por doenças, à drástica diminuição na administração das doses proporciona um acúmulo de suscetíveis e maiores riscos à disseminação das doenças imunopreveníveis. Ressalta-se que, a vacina Hepatite B, a vacina Bacille Calmette - Guerin (BCG) é aplicada ao nascer e também se encontra em desabastecimento em diversos estados, portanto, fatos importantes umas vez que impossibilita a vacinação oportuna e interferem a não administração e/ou interrupção do esquema vacinal.

Seguindo aos desafios dispostos as adequações das coberturas, atualmente, os benefícios das vacinas estão constantemente sendo alvo de crítica por grupos anti-vacina, os quais utilizam os recursos tecnológicos, devido à superficialidade de conteúdo, para disseminação de informações sem base científica, as chamadas "Fake News" questionando a obrigatoriedade, segurança e os esquemas vacinais, afetando significativa sua credibilidade e confiança (Shimizu, 2018; Chaves et al., 2020).

Destarte, considerando o contexto pandêmico da Covid-19, as atuações dos grupos anti-vacina tem ganhado notoriedade e, segundo Conselho Nacional de Secretaria em Saúde (Conass, 2021), um quantitativo significativo de "Fake News" foram registradas, com destaque: a vacina contra a Covid-19 pode provocar alterações genéticas ou câncer, contudo apesar de desmitificadas, essas informações interferem na credibilidade das vacinas e detém influência aos demais 
imunobiológicos distribuídos de forma gratuita pelo SUS, a exemplo a relação da tríplice viral com autismo, HPV e paralisia, e a vacina da poliomielite e a síndrome da morte súbita do lactente (SMSL), despotencializando o PNI e se tornando um problema de saúde pública (Cruz, 2017; Metrópoles, 2020; Chaves et al., 2020; 2021).

Diante disso, estudos recentes avaliam o grau de confiança e aceitabilidade das vacinas, segundo Larson et al., 2015; 2016 em 67 países, a média de desconfiança na segurança da vacina foi de 13\%, com maior grau na frança, com 41\%; para Research América e pela American Society of Microbiology (2018), o Norte Americado expõem uma menor confiança na vacinação ao comparar com 10 anos atrás, de $77 \%$ contra $85 \%$. No mais, a nível de Brasil, $15 \%$ não são confiantes na segurança e efetividade da vacina e, em alguns casos, questionam o quantitativo de doses necessárias para prevenção. A partir desses dados, corroboram a falta de credibilidade nas vacinas em nível global e, com isso, os desafios dispostos ao controle e prevenção de doença, além da enorme influência das informações sem base científica à hesitação vacinal. Contudo, independente dos fatores à hesitação, a vacinação é de caráter social e coletivo do ponto de vista ético-deontológico e humanizado, sendo responsabilidade sanitária de todos e importante fator de qualidade da saúde pública.

Nesse contexto, como instrumento base no combate aos grupos anti-vacina, é crucial o fortalecimento e qualificação de estratégias que visem à ampliação e circulação em massa das informações de qualidade e condizentes com a real contribuição das vacinas, com intuito de incentivar a imunização e aumentar a confiança do público diante a seguridade das vacinas e então estabelecer o principal objetivo do PNI, manter adequas as coberturas vacinais e, consequentemente, o controle das doenças (Cruz, 2017; Chaves et al., 2020; 2021; Conass, 2021).

Segundo Pessoa e Moraes (2019), o Brasil é considerado de baixa prevalência de infecção pelo VHB, contudo, apresenta áreas em que a prevalência se mantem alta, chegando a mais de 8\%, como exemplo na região Amazônia e, sendo a vacina disposta em postos de saúde há mais de três décadas, de forma gratuita e para qualquer faixa etária, a alta prevalência na Amazônia reflete a não adesão à principal forma de prevenção. O Ministério da Saúde estabelece calendário de vacinação por ciclo de vida: infantil, adolescentes, adultos/gestantes e idosos, com hepatite B inclusa em todos, todavia, de acordo com Conselho Federal de Enfermagem - Cofen (2018), Sampaio et al (2019), Veiga et al (2019) e Chaves et al (2020), encontramse abaixo do preconizado em todas as faixas, corroborando o perfil de susceptibilidade desses indivíduos. Ademais, segundo Francisco et al (2015) e Senson et al (2018) a população mais exposta ao VHB são indivíduos de 45 a 49 anos (12,6), 20 a 39 anos (11,6), 50 a 54 anos $(11,8)$ e 10-19 $(1,14)$, dados extremamente preocupantes uma vez que o principal meio de transmissão é por via sexual e, nessas faixas etárias, estão grande parte das mulheres em idade reprodutiva, logo, sujeitas a engravidar.

Portanto, dentro desse público merece destaque a particularidade da transmissão vertical do VHB, que ocorre em cerca de 70\% a 90\% dos casos, sobretudo, devido seu maior risco de cronicidade ao recém-nascido e em crianças de 1 a 5 anos que, segundo Francisco et al (2015) e Silva et al (2020), chega em 90\% e 20 a 50\%, respectivamente, devido a imaturidade do sistema imunológico e caso não seja administrado uma imunoprofilaxia adequada.

Assim, a partir dos dados obtidos pelo Sistema de Informação e Departamento de Informática do SUS de casos confirmados de hepatite B em gestantes/100.000 nascidos vivos, sua distribuição percentual segundo idade gestacional, e o acesso à atenção ao Pré-Natal período de 2010 a 2019, foram observados registrados 2.833 casos durante o período, com maior incidência no RO (2919,6), AC (3734,6), TO (752,7), AM (717,6), RR (704,6), PA (406,3) e AP (195,4) (Figura 2/A). Referente à idade gestacional, AP (50\%), PA (42,7\%) e AM (38,5\%) concentram-se no segundo trimestre, e RO (45,9\%), AC $(45,1 \%)$, RR $(42,3 \%)$ e TO $(41,9 \%)$ no terceiro trimestre. Em nível de assistência ao Pré-Natal, prevalece à categoria "Mais que adequado" em todos os estados, com máxima de $61 \%$ em RO e mínima de 33,7\% no AP, seguido de "Inadequado", máxima no AC (38,9\%) e mínima em RO (20,1\%); “Intermediário", de 16,8\% no AP a 8,8\% em RO; “Adequado", de 10,5\% no PA e TO a 9,2 no RR; de2\% no AP e 0,3 no RO aos que não fizeram assistência (Figura 2/B). 
Figura 2 - Distribuição dos casos confirmados de Hepatite B em gestantes na região Norte, no período de 2010 a 2019. Onde, A Taxa de detecção 100.000 nascidos vivos; B, distribuição percentual segundo idade gestacional e indicador de assistência ao Pré-Natal.

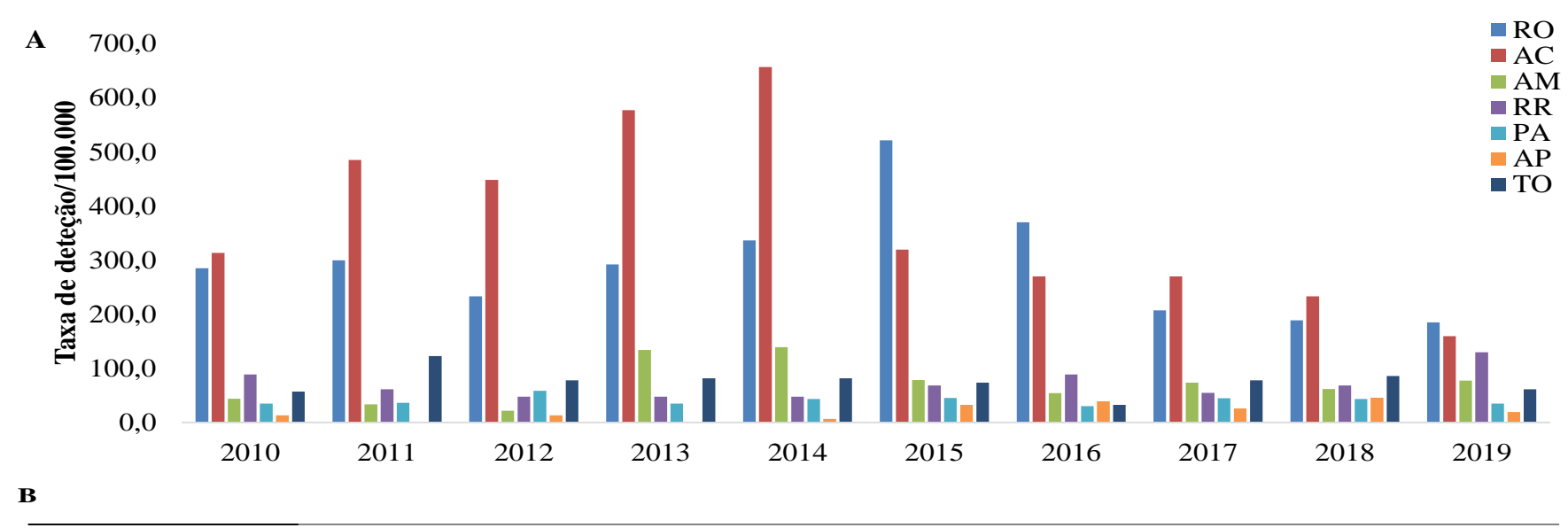

\begin{tabular}{|c|c|c|c|c|c|}
\hline \multirow[t]{2}{*}{ UF/Norte } & \multicolumn{5}{|c|}{$\begin{array}{c}\text { Idade gestacional } \\
(\%)\end{array}$} \\
\hline & $1^{\circ}$ Trimestre & \multicolumn{2}{|c|}{$2^{\circ}$ Trimestre } & \multicolumn{2}{|c|}{$3^{\circ}$ Trimestre } \\
\hline RO & 26,0 & \multicolumn{2}{|r|}{28,1} & \multicolumn{2}{|c|}{45,9} \\
\hline $\mathrm{AC}$ & 17,4 & \multicolumn{2}{|r|}{37,5} & \multicolumn{2}{|c|}{45,1} \\
\hline AM & 24,7 & \multicolumn{2}{|r|}{38,5} & \multicolumn{2}{|c|}{36,8} \\
\hline RR & 33,3 & \multicolumn{2}{|r|}{24,4} & \multicolumn{2}{|c|}{42,3} \\
\hline $\mathbf{P A}$ & 19,1 & \multicolumn{2}{|r|}{42,7} & \multicolumn{2}{|c|}{38,2} \\
\hline AP & 34,6 & \multicolumn{2}{|r|}{50,0} & \multicolumn{2}{|c|}{15,4} \\
\hline \multirow[t]{2}{*}{ TO } & 19,2 & \multicolumn{2}{|r|}{39,0} & \multicolumn{2}{|c|}{41,9} \\
\hline & \multicolumn{5}{|c|}{ Assistência ao Pré-natal } \\
\hline UF/Norte & $\begin{array}{c}\text { Não fez Pré-Natal } \\
(\%)\end{array}$ & $\begin{array}{c}\text { Inadequado } \\
(\%)\end{array}$ & $\begin{array}{c}\text { Intermediário } \\
(\%)\end{array}$ & $\begin{array}{c}\text { Adequado } \\
(\%)\end{array}$ & $\begin{array}{c}\text { Mais que } \\
\text { adequado } \\
(\%)\end{array}$ \\
\hline $\mathrm{RO}$ & 0,3 & 20,1 & 8,8 & 9,7 & 61,0 \\
\hline $\mathrm{AC}$ & 0,9 & 38,9 & 11,3 & 9,6 & 39,3 \\
\hline AM & 1,5 & 37,2 & 12,1 & 9,9 & 39,3 \\
\hline $\mathrm{RR}$ & 0,4 & 38,0 & 12,0 & 9,2 & 40,4 \\
\hline PA & 0,9 & 33,0 & 12,6 & 10,5 & 42,9 \\
\hline AP & 2,0 & 37,4 & 16,8 & 10,1 & 33,7 \\
\hline TO & 0,4 & 21,1 & 9,1 & 10,5 & 59,0 \\
\hline
\end{tabular}

Fonte: Departamento de Informática do Sistema Único de Saúde - DATASUS (atualização dos dados 31/12/2019).

Segundo Sanson et al (2018), devido a alto risco de transmissão e cronicidade ao recém-nascido, além dos riscos predisposto a gestante com HBsAg positivo, a Organização Mundial da Saúde preconiza a realização de teste sorológico para hepatite B no período gestacional como diagnóstico, sobretudo, a primeira consulta ao Pré-Natal e novamente ao terceiro trimestre, para então seguir ao tratamento da gestante e prevenção da transmissão. Cabe ressaltar que, quanto mais precoce for o diagnóstico, consequentemente será o tratamento, logo, mais efetivo serão as intervenções contra transmissão vertical do VHB, todavia, os dados expressos no estudo são de extremo alerta uma vez que, apesar da irregular distribuição percentual da idade gestacional entre os estados, permanece majoritariamente o registro com diagnóstico no terceiro trimestre na região e, além disso, embora a maior taxa esteja em assistência ao Pré-Natal "Mais que adequado", as ocorrências de gestantes que não realizaram a assistência ou encontram-se inadequado ou intermediário expõe o grau de vulnerabilidade à infecção do recémnascido.

Segundo o Ministério da Saúde, o Pré-Natal deve ser iniciado até a $13^{\circ}$ semana de gestação e recomenda o mínimo de 6 consultas, portanto, conforme o índice de Kotelchuck (IK) define-se: Adequado, 6 consultas em idade gestacional <20 semanas; inadequado, menos de 3 consultas iniciado após 20 semanas; intermediário: 3 a 4 consultas durante após 17 semanas 
(Kotelchuck, 1994). Com isso, se estabelece a problemática da baixa adesão à assistência ao Pré-Natal, podendo inferir a detecção tardia ( $3^{\circ}$ trimestre) como o reflexo ao perfil de inadequação aos serviços disponíveis na rede pública, indo a consenso com dados de Sanson et al (2018), onde 43\% das notificações ocorreu no terceiro trimestre e, como destaque, 59,4\% não eram vacinadas e $20 \%$ apresentaram vacinação incompleta. E, embora não avaliado no presente estudo devido a precariedade dos dados específicos a gestantes HBsAg+ pelo DATASUS, diante ao perfil de baixa vacinação em adolescentes e adultos, cogita-se esse mesmo cenário de não imunização das gestantes na região Norte, assim, a importância das adequações à vacinação como medida de prevenção não apenas a gestante, mas a suas crianças, corroborando o caráter coletivo da imunização.

Destarte, Souza et al (2019) detalha em seu estudo que apenas 15,3\% das gestantes realizam o mínimo de 6 consultas e detém assistência adequada, para Sanson et al (2018), apenas 0,1\% realizaram mais de 6 consultas, logo, sendo representativo aos dados desse estudo. Frente a essa precária assistência ao Pré-natal, à literatura traduz fatores que relacionam essa não adesão materna à assistência, sobretudo, ao grau de instrução/escolaridade, restrição de horário e local para consultas, baixa renda, acesso as Unidades Básicas e qualidade da assistência nas redes de saúde (Gonçalves et al., 2017; Cruz et al., 2019; Souza et al., 2019). Souza et al (2019).

Esse carácter assistencial não é restrito as gestantes diagnosticadas com Hepatite B, em estudo recente de Gonçalves et al (2021) sobre a distribuição espacial da sífilis gestacional, uma das principais doenças de risco a transmissão vertical, relatam alta prevalência na região Norte de 2010 a 2019, com maior taxa de detecção ao terceiro trimestre (150,3/100.000hab), prevalente em mulheres com nível fundamental incompleto (134,3/100.000hab), inferindo a isso, a baixa percepção dos riscos da doença durante a gravidez e à transmissão vertical, logo, refletindo em uma diminuição da procura pelos serviços de saúde e o diagnóstico oportuno. Assim como a hepatite B, as medidas de diagnóstico e tratamento precoce são as melhores estratégias de contenção a transmissão vertical, logo, sendo a baixa percepção de risco um fator atrelado tanto para hepatite B quanto à sífilis gestacional, se fundamenta o necessário e crucial o incentivo às práticas diferenciadas com intuito de alcançar populações vulneráveis e então proporcionar informações e orientações acerca da educação em saúde e os serviços necessários durante o período gestacional, fornecendo subsídio para mudança desse perfil tão preocupante.

Ademais, um ponto relatado por Gonçalves et al (2021) é o crescimento concomitante da sífilis gestacional e congênita em áreas com menor cobertura de Estratégia Saúde da Família (ESF). Segunda Valadão et al (2019), a ESF surge como proposta de melhoria aos serviços de saúde na rede de atenção a partir da Política Nacional de Atenção Básica do SUS e, sendo o Pré-Natal uma prática da atenção primária. Essa estratégia emerge como fator positivo ao acompanhamento, prevenção, controle, diagnóstico e tratamento das doenças presentes, sobretudo, no período gestacional, contudo, assim como outros serviços disponibilizados na rede pública, não garantem a universalidade, equidade e a integralidade da atenção, principalmente na região Norte onde apresenta as menores taxa de cobertura da ESF, portanto, implicando na qualidade desses serviços.

Com base dos desafios dispostos e os riscos ao recém-nascido, buscou-se avaliar o perfil da hepatite B na infância, considerando < 1 ano a 14 anos na região Norte, de 2010 a 2018. Logo, a partir dos dados, foi observada maior taxa de detecção em <1 ano em todo período, com somático de 45,5\%/100.000 habitantes, seguido de 10 a 14 anos com 10,8/100.000 habitantes, 5 a 9 anos, 5,3/100.000 habitantes e 1 a 4 anos com 3,9/100.000 habitantes (Figura 3/A). Ademais, considerando as formas clínicas da doença, todas as faixas concentram-se com maior percentagem de hepatite B crônica, sobretudo, em $<1$ ano, com $45,1 \%$ comparado as demais taxas (Figura3/B). 
Figura 3 - Distribuição de casos de hepatite B na infância, na região Norte, no período de 2010 a 2018. Onde, A: Taxa de detecção hepatite B na infância por 100.000 habitantes; B: Percentual da forma clínica da hepatite B segundo faixa etária e forma clínica.

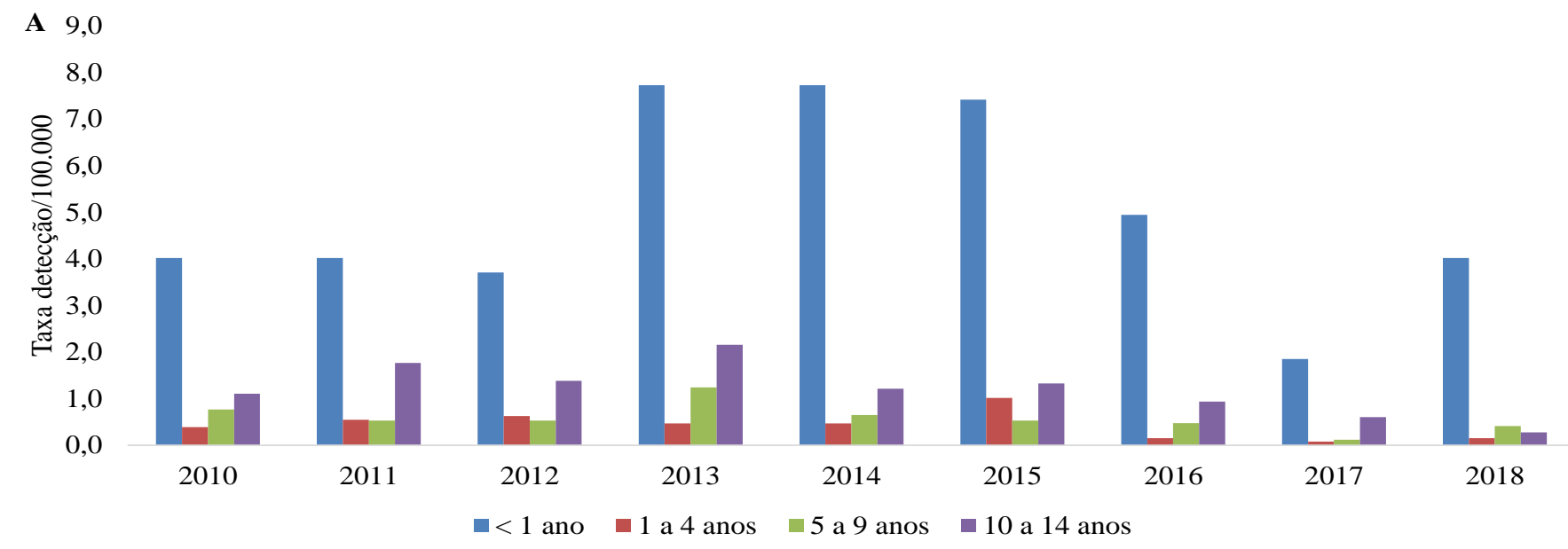

B

\begin{tabular}{ccccccccc}
\hline \multirow{2}{*}{ Ano } & \multicolumn{9}{c}{ Hepatite B Aguda } & \multicolumn{5}{c}{ Hepatite B Crônica } \\
\cline { 2 - 8 } & $<$ 1 ano & $\mathbf{1}$ a 4 anos & $\mathbf{5}$ a 9 anos & $\mathbf{1 0}$ a 14 anos & $<$ 1 ano & $\mathbf{1}$ a 4 anos & $\mathbf{5}$ a 9 anos & $\mathbf{1 0}$ a 14 anos \\
\hline 2010 & 0,6 & 0,1 & 0,4 & 0,1 & 3,4 & 0,3 & 0,5 & 1,0 \\
2011 & 0,9 & 0,0 & 0,2 & 0,3 & 3,4 & 0,6 & 0,7 & 1,5 \\
2012 & 0,3 & 0,1 & 0,2 & 0,3 & 3,4 & 0,5 & 0,4 & 1,1 \\
2013 & 1,9 & 0,3 & 0,2 & 0,5 & 8,3 & 0,5 & 1,5 & 1,9 \\
2014 & 2,2 & 0,2 & 0,3 & 0,6 & 8,7 & 0,5 & 0,6 & 1,2 \\
2015 & 1,5 & 0,4 & 0,1 & 0,2 & 7,1 & 0,9 & 0,6 & 1,3 \\
2016 & 0,6 & 0,1 & 0,2 & 0,2 & 4,6 & 0,1 & 0,2 & 0,8 \\
2017 & 0,6 & 0,0 & 0,0 & 0,1 & 2,2 & 0,2 & 0,1 & 0,6 \\
2018 & 1,2 & 0,2 & 0,4 & 0,0 & 4,0 & 0,2 & 0,2 & 0,3 \\
\hline
\end{tabular}

Fonte: Departamento de Informática do Sistema Único de Saúde - DATASUS (atualização dos dados 31/12/2019).

Sendo público de interesse e de maior destaque, a faixa $<1$ ano possui maior prevalência e maiores taxas de cronicidade, indo a consenso com Ferreira et al (2014), Francisco et al (2015) e Silva et al (2020), onde o público infantil, <1 ano, apresentam geralmente a maior frequência na cronificação da doenças o que pode-se inferir a enorme contribuição de mães que não realizam o Pré-Natal ou apresentam inadequação durante esse processo, refletindo em um tratamento tardio, logo, proporcionando maior a vulnerabilidade na infância e o alto risco predisposto serem portadoras do VHB.

Diante aos esses fatores de risco levantados, a transmissão vertical permanece sendo uma das principais rotas de infeção ao recém-nascido, com isso, uma série de estratégias são disposta com intuito de reduzir as chaves de transmissão. Sali et al (2019), através de uma revisão sistemática e meta-análise, expõe estudos utilizando os métodos de terapia com Lamivudina, Telbivudina e Tenofovir e demonstram que são benéficas e reduzem significativamente a taxa de positividade de HBsAg em recém-nascido, além de ser eficaz quando aplicado no segundo ou terceiro trimestre, contudo, segundo Pessoa e Moraes (2019), deve-se realizar em conjunto a administração de imunoglobulina da hepatite B (HBIg) e vacina da hepatite B para recém-nascidos dentro de 12 horas após o nascimento, logo, seguindo essas orientações, a prevenção da transmissão chega a 95\%. Entretanto, tal eficácia não é 100\% devido a diversos fatores, atraso ou qualidade da terapia da gestante ou nível elevado de HBsAg, durante o parto, atraso na administração da HBIg e a primeira dose da vacina, a falta de incompletude das doses subsequentes. 
A partir do perfil levantado em menores de 1 ano, e a enorme contribuição da HBIg e vacinação logo após o nascimento, pode-se inferir o atrasado na administração da vacina, como exposto anteriormente devido a baixa cobertura vacinal nesse público uma vez que estão incluso nas taxas de cobertura pelo PNI proporcionado, sobretudo, ao desabastecimento dos postos de saúde pela vacina Hepatite B, além disso, assim como a vacina da Hepatite B, segundo MS (2017), os imunoglobulinas anti-hepatite B não foram distribuídos devido a classificação de inadequado pela Anvisa diante ao desvio de temperatura, portanto, reduzindo o estoque em demandas emergenciais. Logo, enaltece os riscos diante as precárias condições na garantia dos insumos à população e sua elevada contribuição ao estabelecimento de infecções em situações com enorme potencial de prevenção, nesse contexto, reforça a necessidade de melhoria dos serviços dispostos nas redes públicas.

\section{Conclusão}

A partir dos dados, foi observada baixa cobertura vacinal tanto para Hepatite B quanto para Pentavalente nas Unidades Federativas da região Norte, assim como a heterogeneidade das coberturas entre os municípios, possibilitando inferir a isso uma série de problemática desde o desabastecimento pontual em postos de vacinação, até a disseminação das Fake News. Ademais, referente ao perfil das gestantes portadoras do VHB, observa-se distribuição em todos os anos e todos os estados, com maior frequência de diagnóstico no terceiro trimestre e com assistência ao Pré-Natal "mais que adequado", contudo, merece destaque as gestantes que não o realizaram ou os fizeram de forma inadequada. Referente ao perfil da hepatite B na infância há maior detecção em menores de um ano, com frequência da forma clínica crônica. Logo, sabendo dos riscos da doença, sobretudo, em menores de 1 ano e, devido à alta probabilidade de se tornar crônico, torna-se essencial fortalecer e incentivar as ações de vacinação promovendo uma política de informação condizentes com as características das vacinas e sua importância, além do aprimoramento da gestão quanto a distribuição dos insumos, proporcionado o aumento da cobertura vacinal em todas as faixas etárias. Além disso, deve-se realizar uma busca ativa de gestantes portadoras do VHB, e orientá-las quanto a uma gestação segura, baseada em assistência de qualidade e o tratamento oportuno, devido ao alto potencial da transmissão vertical, interferindo na qualidade de vida do recém-nascido.

\section{Referências}

Agencia Brasil. (2020). Vacina pentavalente começa a ser distribuída hoje aos estados.

Arroyo, L. H., Ramos, A. C. V., Yamamura, M., Weiller, T. H., Crispim, J. A., Ramos, D. C., Torres, M. F., Santos, D. T., Palha, P. F \& Arcênio, R. A. (2020). Áreas com queda da cobertura vacinal para BCG, poliomielite e tríplice viral no Brasil (2006-2016): mapas da heterogeneidade regional. Ad. Saúde Pública: 36(4): e00015619.

Brasil. (2012). Ministério da Saúde. Informe Técnico Da Introdução Da Vacina Pentavalente Vacina adsorvida difteria, tétano, pertussis, hepatite B (recombinante) e Haemophilus influenzae tipo b (conjugada). DF.

Brasil. (2020). Ministério da Saúde. Coordenação Geral do Programa Nacional de Imunizações. Calendário Nacional de Vacinação.

Brasil. (2020). Ministério da Saúde. Boletim Epidemiológico das Hepatites Virais.

Castro, A. A. M., Bernardo, A. L. B., Ventura, M. L. A., Veras, A. A. C. A., Caminha, M. F. C., Silva, M. B., Silva, S. L \& Lima, E. J. F. (2018). Fatores associados à cobertura vacinal em crianças: análise de uma comunidade. Faculdade Pernambucana de Saúde, Recife-PE.

Césare, N. (2020). O perfil longitudinal da cobertura vacinal no Brasil revela uma mudança recente nos padrões caracterizados pela redução diferencial entre as regiões. International Journal of Infectious Diseases, 98: 275-280.

Chaves, E. C. R., Júnior, K. N. T., Furlaneto, I, P., Aarão, T. L. S., \& Mendonça, M. H. R. (2020). Avaliação da situação vacinal e a percepção de acadêmicos dos cursos da área da saúde de um centro universitário particular de uma cidade do estado do Pará. Revista Eletrônica Acervo Saúde, 12 (11), e4705.

Chaves, E. C. R., Júnior, K. N. T., Andrade, B. F. F \& de Mendonça, M. H. R. (2020). Avaliação da cobertura vacinal do sarampo no período de 2013-2019 e sua relação com a reemergência no Brasil. Revista Eletrônica Acervo Saúde, (38), e1982.

Chaves, E. C. R., Júnior, K. N. T., Santos, B. P. S., Oliveira, D. J. S., Palmeira, A. B., Pereira, D. F. P., Aarão, T. L. S., Furlaneto, I. P. F., Lima, S. B. A \& Mendonça, M. H. R. (2021). Os impactos da variante P1 (501Y, V3) do Sars CoV-2, na Amazônia. International Journal of Development Research, 11(5); 46748-46754. 
Conselho Federal de Enfermagem - Cofen. (2018). Todas as vacinas do calendário de adultos estão abaixo da meta de cobertura ideal, G1.

Cruz, A. (2017). A queda da imunização no Brasil. Consensus.

Dias, B. F \& Almeida, R. M. V. R. (2017). Rotavirus and Triple Viral Vaccine Losses in Vaccination Rooms: a Cross-Sectional Study. MedicalExpress, 4(4).

Cruz, G. C., Ruiz, P. C., Júnior, O. C. R., Sousa, A. D., Pereira, R. M. O., Barroso, C. O \& Campos, S. S. T. (2019). Métodos de avaliação da qualidade de assistência ao pré-natal no Brasil: revisão integrativa da literatura. Revista Eletrônica Acervo Saúde, $27: \mathrm{e} 521$

Gonçalves, N., Miranda, C. S. C., Guedes, J. A., Silva, L. C. T., Barros, E. M., Tavares, C. G.M., Palácios, V. G. C. M., Costa, S. B. N \& Xavier, M. B. (2019). Hepatites B e C nas áreas de três Centros Regionais de Saúde do Estado do Pará, Brasil: uma análise espacial, epidemiológica e socioeconômica. Cad. Saúde Colet., 27 (1): 1-10.

Gonçalves, D. G. N., Mezzomo, E. S., Carvalho, J. R., Souza, L. C. K. \& Mendona, M. H. R. (2021). Avaliação da distribuição espacial da sífilis gestacional e congênita na região Norte e Nordeste. International Journal of Development Research, 11, (05): 46916-46922.

Ferreira, A. R., Fagundes, E. D. T., Queiroz, T. C. N \& Júnior, R. C. N. (2014). Hepatites Virais A, B e C em crianças e adolescentes. Rev Med. Minas Gerais, $24(2)$.

Fonseca, K. R \& Buenafuente, S. M. F. (2020). Análise das coberturas vacinais de crianças menores de um ano em Roraima, 2013-2017. Epidemiol. Serv. Saúde: 18.

Francisco, P. M. S. B., Donalisio, M. R., Gabriel, F. J. O \& Barros, M. B. A. (2015). Vacinação contra hepatite B em adolescentes residentes em Campinas, São Paulo, Brasil. Rev Bras Epidemiol, 18(3): 552-567.

Fundação Oswaldo Cruz -Fiocruz. (2020). Vacinas e vacinação no Brasil: horizontes para os próximos 20 anos.

Kotelchuck, M. (1994). An evaluation of Kessner adequacy of prenatal care index and a proposed adequacy of prenatal care utilization index. Am J Public Health, 84:1414-20.

Larson, H. J., Jerrett, C., Schulz, W. S., Chaudhuri, M., Zhou, Y., Dube, E., Schuster, M., MacDonaldo, N. E \& Wilson, R. (2015). Measuring vaccine hesitancy: the development of a survey tool. Vaccine, 33(34):4165-75.

Larson, H. J., Figueiredo, A., Xiahong, Z., Schulz, W. S., Verger, P., Johston, I. G., Cook, A. R \& Jones, N. S. 1. (2016). The state of vaccine confidence 2016: global insights through a 67-country survey. EBioMedicine, 12:295-301.

Macedo, L. M., Gomes, M. M., Madureira, M. L. L., Lemos, N. B., Lucinda, L. M. F \& Araújo, S. T. H. (2017). Atraso vacinal no município de Barbacena (MG) contextualizando o problema. Revista Interdisciplinar de Estudos Experimentais, 9: 7-14.

Maciel, J. A. P., Silva, A. C, Campos, J. S., Correia, L. L., Rocha, H. A. L., Rocha, S. G. M. O \& Sampaios, E. M. (2019). Análise do estado de cobertura vacinal de crianças menores de três anos no município de Fortaleza em 2017. Rev Bras Med Fam Comunidade, 14(41): 1824.

Metrópoles. (2020). Entidade alerta para avanço das fake news sobre vacina: Opas alerta que falsas notícias circulam em redes sociais e aplicativos de mensagens, desencorajando as pessoas a se vacinarem. Brasília, DF: Metrópoles.

Nóvoa, T. A., Cordovil, V. R., Pantoja, G. M., Ribeiro, M. E. S., Cunha, A. C. S., Bendamin, A. I. M., Silva, C. D. C. C \& Silva, T. N. (2020). Cobertura vacinal do programa nacional de imunizações (PNI). Braz. J. Heat. Rev., Curitiba, 3(4): 7863-7873.

Pessoa, M. G \& Moraes, A. (2019). Hepatites virais na gravidez. Femina, 47(1): 37-41.

Pinheiro, L. (2020). Produção nacional de medicamentos pode evitar desabastecimento de vacinas. Inesc.

Pinheiro, L. (2020). Metade das crianças brasileiras não recebeu todas as vacinas que deveria em 2020, apontam dados do Ministério da Saúde. G1, São Paulo.

Prado, M. (2015). Falta de abastecimento de vacinas prejudica estoques em Blumenau. Prefeitura de Blumenau.

Research America. (2018). Americans' views on vaccines and infectious diseases. Arlington: Research Americ.

Sali, S., Darvishi, M., GhasemiAdl, M., Akhalaghdoust, M., Mizazadeh, A., Behjati, S. E., Zeinolabedini, H. S., Shokouh, S \& Tavakolpour, S. (2019). Comparing the Efficacy and Safety of Treating Chronic Hepatitis B Infection during Pregnancy with Lamivudine, Telbivudine, and Tenofovir: A Metaanalysis. Journal of Clinical and Translational Hepatology, 7(3):197-212.

Sanson, M. C. G., Feitoza, H. A. C., Saraceni, V., Koifman, R. J \& Bessa, A. R. S. (2018). Prevalência e perfil epidemiológico da Hepatite B em gestantes: um estudo populacional em uma cidade da Amazônia Ocidental brasileira, no período de 2007 a 2015. Rev. Bras. Saúde Mater. Infant., Recife, 18 (4): $723-733$.

Sampaio, S. M. F., F. C., Oliveira, P. P., Lanza, F. M., Oliveira, V. C \& Santos, W. J. (2019). A vacinação e o saber do adolescente: educação em saúde e ações para a imunoprevenção. Ciência \& Saúde Coletiva, 24(2): 351-360.

Silva, S., Batista, S. J \& Silva, M. E. D. C. (2020). Fatores associados ao descumprimento do calendário vacinal de crianças até 05 anos. Bioética e Saúde Pública, 1(29): 291-309.

Silva, F. S., Barbosa, Y. C., Batalha, M. A., Ribeiro, M. R. C., Simões, V. M. F \& Branco, M. R. F. C. (2018). Incompletude vacinal infantil de vacinas novas e antigas e fatores associados: coorte de nascimento BRISA, São Luís, Nordeste, Brasil. Cad Saude Publica, 34(3):e00041717

Silveira, M. F., Buffarini, R., Bertoldi, A. D., Santos, I. S., Barros, A. J. D., Matijasvich, A., Menezes, A. M. B., Gonçalves, H., Horta, B. L., Barros, F. C., Barata, R. B \& Victoria, C. G. (2020). The emergence of vaccine hesitancy among upper-class Brazilians: Results from four birth cohorts, 1982-2015. Vaccine, 38(3):482-488. 
Research, Society and Development, v. 10, n. 8, e25510817027, 2021

(CC BY 4.0) | ISSN 2525-3409 | DOI: http://dx.doi.org/10.33448/rsd-v10i8.17027

Shimizu, N. R. (2018). Movimento Antivacina: A Memória Funcionando No/Pelo (Per) Curso Dos Sentidos e dos Sujeitos na Sociedade, Revista do Edicc, $5(1)$.

Sociedade Brasileira de Imunização - SBIm. (2015). Desabastecimento das vacinas combinadas à DTPa — Novidades sobre disponibilização da quíntupla do PNI para os reforços. São Paulo.

Sociedade Brasileira de Pediatria - SBP. (2020). Desabastecimento de vacinas nos postos públicos de vacinação do país.

Sociedade Brasileira de Pediatria - SBP. (2020). Manifesto pela saúde das crianças brasileiras: O País precisa aumentar sua cobertura vacina.Sociedade

Brasileira de Imunização - SBIm. (2020). Vacina da hepatite B

Sociedade Brasileira de Imunização - SBIm. (2020). Vacina Pentavalente.

Souza, L (2020). A vacina pentavalente começa a ser distribuída aos estados. Agencia Brasil.

Souza, I. A., Serinolli, M. I \& Novaretti, M. C. Z. (2019). Assistência pré-natal e puerperal e indicadores de gravidade no parto: um estudo sobre as informações disponíveis no cartão da gestante. Rev. Bras. Saúde Mater. Infant., Recife, 19 (4): 991-997

Tauil, M. C., Sato, A. P. S., Costa, A, A, C., Inemani, M., Ferreira, V. L. R \& Waldmn, E. A. (2017) Coberturas vacinais por doses recebidas e oportunas com base em um registro informatizado de imunização, Araraquara-SP, Brasil, 2012-2014*. Epidemiol. Serv. Saúde, Brasília, 26(4): 835-846.

Valadão, P. A. S., Lins, L \& Carvalho, F. M. (2019). Melhor no passado: a verdadeira saúde da família. Saúde soc., 28(1).

Wolkers, P. C. B., Santos, J. S., Yakuwa, M. S., Pina, J. C., Furtado, M. C. C \& Mello, D. F. (2016) O direito à imunização na infância e adolescência: uma revisão narrativa. Ciência Y Enfermeria XXII (3): 85-96.

Organização Mundial da Saúde - OMS. (2014). Relatório do grupo de trabalho Sage sobre hesitação vacinal. Geneva. Zorzetto.

Organização Mundial da Saúde - OMS (2018). Hepatite B. Genebra. 